\title{
Exploration on Security System Structure of Smart Campus Based on Cloud Computing
}

\author{
Wei Zhou \\ Information Center, Shanghai University of Engineering Science, Shanghai, China
}

Keywords: Smart campus. Cloud computing. Campus cloud. Security system structure

\begin{abstract}
The construction of security system structure of smart campus is one of the core problems restricting the development of smart campus. This paper studies such problems as smart campus, smart campus system structure and campus cloud structure from the perspective of information security, designs security system structure of smart campus on this basis and describes the security system structure at six levels.
\end{abstract}

\section{Introduction}

Currently, most colleges and universities have campus network. The campus network of some colleges and universities has a substantial scale. However, network resource sharing of colleges and universities generally has limitations. As different departments often use different operating environments and software systems, it is difficult to realize resource sharing and resource utilization and allocation are not coordinating. Under this background, many colleges and universities put forward the objective of building smart campus. Smart campus is the latest manifestation of informatization campus. It uses computer technology, network technology and communication technology to conduct comprehensive informatization management over resources related to teaching, scientific research, management and life in schools. However, the higher the degree of informatization of campus network is, the more unexpected information security will be. Therefore, how to establish security system structure of smart campus based on cloud computing and guarantee the security of smart campus is an important issue in the research on smart campus network and its application system currently.

\section{System structure of smart campus}

Table 1. System structure of smart campus

\begin{tabular}{|c|c|c|}
\hline \multirow{5}{*}{$\begin{array}{l}\text { Information } \\
\text { standard } \\
\text { system }\end{array}$} & Information service layer & \multirow{5}{*}{$\begin{array}{l}\text { Security } \\
\text { protection } \\
\text { architectur } \\
\mathrm{e}\end{array}$} \\
\hline & Service application layer & \\
\hline & Service supporting layer & \\
\hline & Basic data layer & \\
\hline & Network physical layer & \\
\hline
\end{tabular}

People-oriented concept is one of the important concepts of modern schools. How to reflect people-oriented concept in the construction process of smart campus is the key to the success of the construction and administrators' macro understanding and mastery of smart campus from the overall perspective of campus informatization construction. We establish the system structure of smart campus with this starting point, as shown in table 1. Information service layer provides unified system access service for all service users. Service application layer includes various specific business processing and information management application system provided for users. Service supporting layer provides various services such as data storage, access and sharing for layers above. Network physical layer provides information access and data communication for four layers above and the integration of physical network. 


\section{Campus cloud}

Cloud computing is not a brand new technology. It develops based on parallel computing, grid computing, distributed computing and virtualization. It is a new development model of these concepts. This model integrates many idle computing resources in the same place or in different places and provides products, services and solutions for users or enterprises through network. With the gradual development and popularization of cloud computing model, information processing of schools, educational institutions and individuals will gradually move to cloud. This will produce positive influence on the application of campus network.

Campus cloud needs to meet the need of application and user scale growth. Campus cloud takes such measures as data multi-copy fault tolerance and computational node isomorphism and interconvertibility to guarantee the high reliability of data. The greatest advantage of campus cloud for the provision of various information requirements consists in thin terminal. Using virtualization technology to integrate various resources, provide a great pool of resources and make dynamic adjustment can significantly improve the utilization rate of resources. We can use cloud computing and virtualization technology to integrate existing resources, realize complicated scientific calculation, experimental research, massive data mining and large-scale distributed storage under the premise of not inputting large equipment, make dynamic adjustment of resources and realize the performance of super computer.

Cloud computing platform can provide multiple service forms, such as SaaS, DaaS, PaaS, IaaS and CaaS. Campus cloud is a typical cloud architecture. Basic technologies mainly include Service-Oriented Architecture and virtualization. Generally, campus cloud can be divided into three basic layers: Infrastructure, Platform and Application. Infrastructure layer has large-scale hardware resources. These hardware facilities are connected with each other through campus network. To realize high-level resource management logic, it is required to virtualize hardware resources. On the one hand, virtualization process shields differences of hardware products; on the other hand, it provides unified management logic and interface for each hardware resource. Platform layer provides rich "cloud middleware" resources for users. These resources include the environment during operation, sharing service and automated management service etc. Application layer provides users with simplified distributed software development, test and deployment environment and customized software application.

\section{Security system structure of smart campus}

While bringing convenience to smart campus, campus cloud also faces a series of security problems. Besides the long-existing information security problem, there are also new security problems caused by cloud computing, such as scattered storage of data under the environment of cloud computing and openness of cloud service. All this brings new challenges to the existing security system. Its security concerns whether cloud computing can be applied in smart campus. According to the practical situation in combination with characteristics and technologies of cloud computing, corresponding to cloud computing architecture, security system structure of smart campus contain six layers, i.e. physical security, network security, data security, service security, application security and management security, as shown in fig.1.

\section{Physical security}

Physical security is the basis and premise of guaranteeing safe operation of smart campus. This paper studies ho to improve physical security mainly in three aspects - environment, equipment and medium. (1) Environmental security. Site facilities such as machine room, computer equipment and monitoring and ambient environment and fire safety should meet relevant national standards; machine room should keep away from various high risk (earthquake, magnetic field, lightning and fire disaster) regions; when the system is damaged, it should have corresponding forecasting, alarm and automatic damage elimination mechanism; the system should not only have complete fault tolerance measures and single-point fault correcting measure; to prevent electromagnetic leakage, 
internal equipment of the system should use technologies such as shielding and ant-interference. (2) Equipment security. To guarantee safe operation of system equipment, power and electrostatic protection technologies, anti-virus, anti-electromagnetic, anti-short circuit/open circuit technologies etc. should be used. Equipment operators should receive corresponding education, training and management. There should be a reasonable man-machine communication mechanism so as to avoid abnormal operation of equipment to a great extent and improve the efficiency and lifetime of equipment. (3) Medium security. When the use of transmission medium of smart campus network is considered, optical fiber should be used. It is sealed in plastics and placed underground, which can prevent open circuit/short circuit and parallel wiretap.

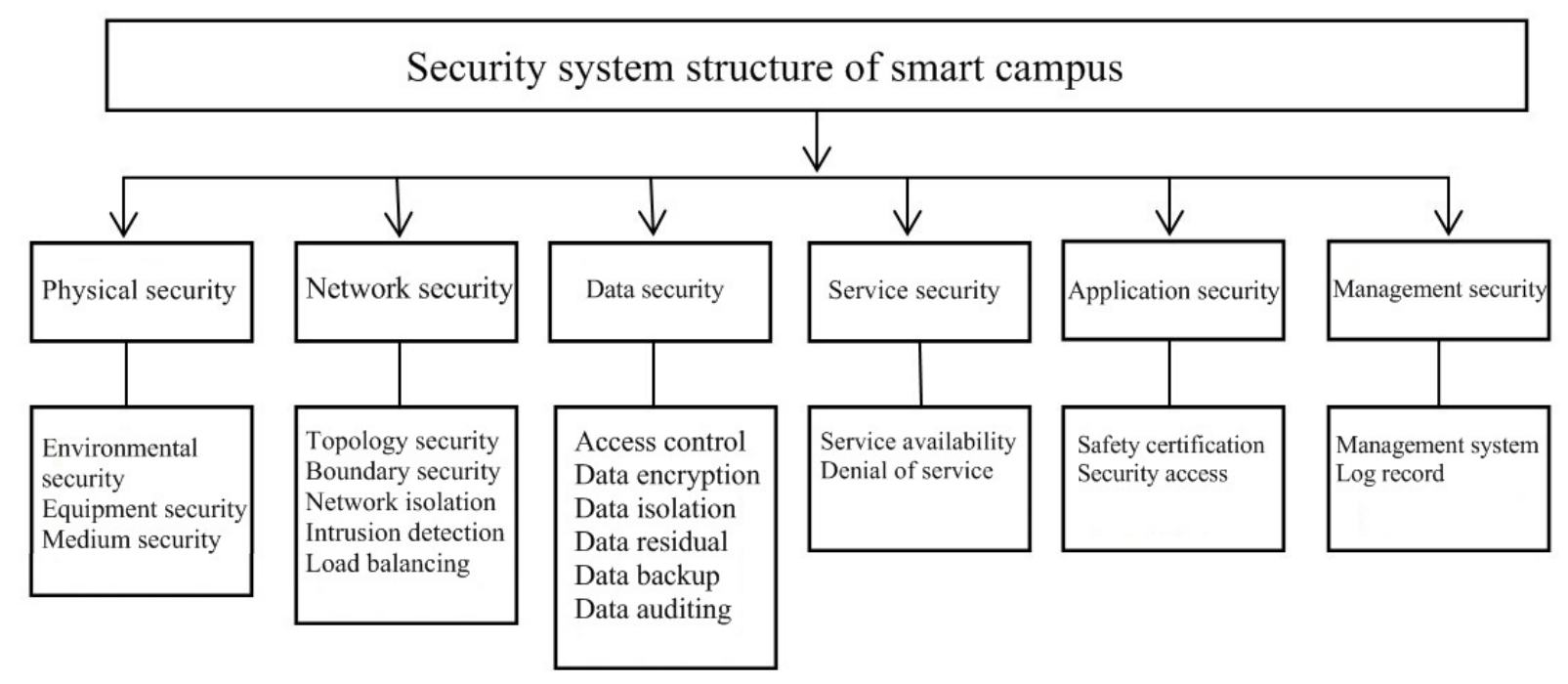

\section{Network security}

Fig. 1. Security system structure of smart campus

All applications of smart campus provide services based on network. Therefore, the reliability of network security of campus cloud becomes the key to normal and continuous service provision. Network security is guaranteed mainly in such aspects as network topology security, partition of security domain and boundary protection, data flow load balancing, access control of network resources, means of intrusion detection and network facility anti-virus.

Network topology security uses new network topological structure which enables higher connectivity of nodes and fault tolerance ability and easier load balancing. In campus cloud, physical security boundary gradually disappears and is replaced by logic security boundary. Virtual private network and data encryption technologies should be adopted to ensure transmission security of user data. To prevent excessive concentration of data flow which causes network congestion, campus cloud system should be able to analyze the load level of server, correctly guide user requests according to load level, automatically choose those stable and reliable networks and realize load balancing between servers. In cloud computing data center, such technologies as VLAN and distributed virtual switch are adopted to realize isolation between user systems. Distribution intrusion detection and virus protection system are used to resist attack from off-campus network. In addition, other security measures and technologies can be adopted, such as port binding and establishment of virtual firewall.

\section{Data security}

Data center is the center of virtualization and efficient management of cloud computing so as to achieve the purpose of centralized management, flexible access and data security. As data are stored in cloud computing platform and security threats faced by them are more prominent. According to data information security standard requirements under the environment of cloud computing, technical 
means should be taken in such aspects as data access control, data encryption, data isolation, data residual, data backup and data audit to ensure the security of data in campus cloud.

\section{Access control}

Different data have different permissions. To maintain the privacy of data, only users with permission can access data. Campus cloud can establish unified identity management, security certificate and access permission control and use authentication technologies such as digital signature, single sign on authentication and double-factor login authentication to ensure data security.

\section{Data encryption}

Data encryption is an important method to ensure data privacy. Important sensitive data are encrypted automatically. Even if they are filched by illegal users, it is unnecessary to worry about data leakage. Symmetric encryption algorithm with a high encryption performance should be selected for encryption transmission and storage of data. In addition, it is necessary to encryption file system. The robustness of encryption algorithm depends on good key management technology. Meanwhile, key management is the premise of realizing user identity identification and authentication.

\section{Data isolation}

The establishment of software system of campus cloud widely uses single software system case to serve multiple customers. This means that data of all users are in the sharing environment under the multi-tenant environment. Therefore, it is necessary to develop an extra data isolation mechanism to ensure invisibility of data among customers. Currently, several mature structures are used to help the system realize data isolation, including Shared Schema Multi-Tenancy, Separated Database and Shared Database Separated Schema. In addition, physical isolation and virtualization can be used to realize security isolation of data and configuration information among different tenants.

\section{Data residual}

In campus cloud, data residual means that data remain after wiped in certain form. Some physical characteristics might remain after data are wiped so that data can be reconstructed. Therefore, data residual might make some sensitive information leaked unconsciously. Therefore, before reallocation of storage resources, complete removal of data can be ensured with such technologies and methods as destruction of relevant media of enciphered data, destruction of storage medium, disk wiping and content discovery so as to avoid illegal reconstruction of data.

\section{Data backup and restoration}

When data are stored in campus cloud, users will worry about the loss of data. To ensure absolute security of user data and cope with data loss and business cease caused by emergency and extreme case, campus cloud should be able to implement disaster recovery plan rapidly and continue to provide services. Therefore, it is required to improve disaster recovery backup mechanism of smart campus and improve the robustness of cloud computing system.

\section{Data auditing}

To ensure the accuracy and effectiveness of data, a third-party certification authority is often introduced for data auditing. An independent third party conducts monitoring and evaluation of business users and provides security auditing report specific to operation and maintenance management of cloud computing supplier. This is essential to ensure cloud computing service.

\section{Service security}

Safe smart campus should allow authorized users to use cloud computing service and can still provide effective services for authorized users when the system is damaged partially or it is required to degrade for use. To ensure the demand of the system for availability, cloud computing system can introduce such mechanisms as identification and authentication, access control, data flow control and audit. Due to intensive concentration of users and information resources, it easily becomes the target of hacker attack. Meanwhile, as the consequence and destructive effect caused by denial of service attack will obviously exceed traditional network application environment, we can strengthen the security of host, restrict communication channel with poor foundation and adjust firewall and rules of intrusion detection system to ensure service security.

\section{Application security}


It is required to establish strict cloud computing AAA mechanism through identity authentication and security access control, implement strict identity management, security authentication and access permission control, provide user access records, realize traceability of access and ensure the security of user application.

\section{Management security}

Currently, most safety accidents in the network are caused by internal workers of network. Therefore, to realize the security of system structure of smart campus, administrative management is extremely important for the realization of security besides the guarantee of technical security. It is required to establish security management system and security auditing system according to practical situation of the school and record and maintain various security log contents so as to standardize the operation and behaviors of administrative staff and improve post review ability of illegal trace to the source.

\section{Conclusion}

Security system structure of smart campus network determines the success or failure of construction of smart campus and is one of the core problems restricting the development of smart campus. Security system structure of smart campus put forward in this paper describes security structure problems at six layers and provides strong security guarantee for the construction of smart campus. However, with the construction and development of smart campus, new security problems will occur constantly and inevitably make new requirements for security system structure of smart campus. The further design and improvement of security system structure of smart campus will become an impetus of the construction of smart campus and promote the development and progress of informatization of colleges and universities in China.

\section{Acknowledgments}

This paper is the sub-topic: "Research on Large-scale High-speed, Safe and Real-time Industrial Network of High-end Large-scale Programmable Automation System" of the topic of national high-tech research development plan (863 plan): Development and Application of High-end Large-scale PLC Programmable Automation System, No.: 2013AA040302.

\section{References}

[1] Lei Wanyun: Cloud Computing Technology, Platform and Application Case, Beijing: Tsinghua University Press, 2011.

[2] Wang Peng: Key Technologies and Application Case of Cloud Computing, Beijing: Posts and Telecom Press, 2010.

[3] Bo Mingxia, Chen Jun: Research on Security System Structure of Cloud Computing, Information Network Security, 2011.8.

[4] Lv Qian: Establishment of Smart Campus Based on Cloud Computing and Internet of Things, Computer Science, 2011,38(10):18-21.

[5] Bo Mingxia, Chen Jun: Research on Security System Structure of Cloud Computing, Information Network Security, 2011.8.

[6] Li Zheng, Wang Lu: Research on Application of Cloud Computing in Smart Campus, Computer and Modernization. 2012(201):48-50, 